\title{
A Prospective Study of Clinical and Radiological Outcomes of Zero-Profile Cage Screw Implants for Single-Level Anterior Cervical Discectomy and Fusion: Is Segmental Lordosis Maintained at 2 Years?
}

\author{
Saumyajit Basu ${ }^{1}$, Sreeramalingam Rathinavelu ${ }^{2}$ \\ ${ }^{1}$ Park Clinic, Kolkata, India \\ ${ }^{2}$ Ortho One Orthopaedic Subspecialty Centre, Coimbatore, India
}

\begin{abstract}
Study Design: Prospective cohort study.
Purpose: To study clinicoradiological parameters of zero-profile cage screw used for anterior cervical discectomy and fusion (ACDF). Overview of Literature: Radiological parameters of various implants used for ACDF are available, but those for zero-profile cage are sparse.

Methods: Patients with unilateral intractable brachialgia due to single-level cervical disc prolapse between April 1, 2011 and March 31, 2014 were included. Clinical assessment included arm and neck pain using visual analogue score (VAS) and neck disability index (NDI) scores. Radiological assessment included motion segment height, adjacent disc height (upper and lower), segmental and cervical lordosis, implant subsidence, and pseudoarthrosis. Follow-ups were scheduled at 1, 3, 6, 12, and 24 months.

Results: Thirty-four patients (26 males, 8 females) aged 30-50 years (mean, 42.2) showed excellent clinical improvement based on VAS scores (7.4-0 for arm and 2.0-0.6 for neck pains). Postoperative disc height improved by $11.33 \%(p<0.001)$, but at 2 years, the score deteriorated by $7.03 \%(p<0.001)$. Difference in the adjacent segment disc height at 2 years was $0.08 \%(p=0.8)$ in upper and $0.16 \%$ $(p<0.001)$ in lower disc spaces. Average segmental lordosis achieved was $5.59^{\circ}(p<0.001)$ from a preoperative kyphosis of $0.88^{\circ}$; at 2 years, an average loss of $7.05^{\circ}(p<0.001)$ occurred, resulting in an average segmental kyphosis of $1.38^{\circ}$. Cervical lordosis improved from $11.59^{\circ}$ to $14.88^{\circ}(p=0.164)$, and at 2 years, it progressively improved to $22.59^{\circ}(p<0.001)$. Three patients showed bone formation and two mild protrusion of the implant at 2 years without pseudoarthrosis/implant failure.

Conclusions: The zero-profile cage screw device provides good fusion and cervical lordosis but is incapable of maintaining the segmental lordosis achieved up to a 2-year follow-up. We also recommend caution when using it in patients with small vertebrae.
\end{abstract}

Keywords: Zero profile; Cage screw; Anterior cervical discectomy and fusion; Lordosis; Anterior cervical discectomy

\section{Introduction}

The most common surgery performed for cervical radicu- lopathy is anterior cervical discectomy and fusion (ACDF). Except for the fact that good decompression is a must for good results, other aspects of this surgery such as fusion,

Received Apr 14, 2016; Revised Sep 11, 2016; Accepted Sep 27, 2016

Corresponding author: Saumyajit Basu

Park Clinic, 4, Gorky Terrace, Kolkata 700017, India

Tel: +913322801986, E-mail: saumyajit2016@gmail.com 
motion preservation, and implant of choice are controversial. ACDF is considered the gold standard treatment in elderly patients and those with contraindications for a cervical disc replacement [1]. Anterior cervical plates are commonly used to achieve stabilization; however, dysphagia, possibly related to profile of the plate, adjacent segment disease, and inherent implant-related problems are the associated complication. A cage screw device that provides immediate biomechanical stability as good as that provided by an anterior cervical plate has been introduced (e.g., Zero-P by DePuy Synthes).

These implants have a locking plate-screw interface, which holds the screw cage construct stable. The plate has an internal screw thread that engages with the outer screw thread located in the head of the screw. This makes the implant constrained and gives an angle-stable screw fixation. Short-term studies on such zero-profile implants are now being conducted, most of which report that complications such as dysphagia and implant failure associated with the plates are less frequent with these newer implants and that the implants have a fusion rate similar to the plates.

We conducted a prospective study on patients who underwent ACDF for single-level disc pathology after an adequate period of conservative trial. None of the authors have any association with or received any financial assistance for this work from any commercial medical industry. The literature available to date discusses only the clinical outcome of these implants. We have studied the radiological outcome by resolving the following questions: Is it possible to achieve and maintain normal segmental and cervical lordosis? Does any significant implant subsidence occur? What is the impact on adjacent segments? Do any other radiological findings have an impact on long-term outcome?

\section{Materials and Methods}

This is a prospective study. Patients $(n=37)$ were recruited from January 2010 to December 2011. Inclusion criteria were presentation with unilateral upper limb radiculopathy due to single-level disc prolapse in the subaxial cervical spine and aged 20-60 years. All the patients underwent conservative treatment for $\geq 4$ weeks. This time period was chosen as it allowed most of our patients to return to work after reasonable leave duration without adequate pain relief [2]. Those who presented with myelopa- thy, predominant neck pain, congenital anomalies in the cervical spine, spondyloarthropathies, DISH, metabolic disorders, and radiculopathy due to other pathologies and those with comorbidities likely to influence the outcome were excluded from this study. Patients were followed up for 2 years. Data analysis was started after the last recruited patient's 2-year follow-up was performed. The 2-year follow-up was considered as the end point of this study; hence, further follow-up data were not included.

Clinical assessment included the neck disability index and visual analogue score (VAS) scores for radiculopathy. All the patients were made to enter VAS scores in the preoperative period and then at follow-up visits. VAS scores were noted in the preoperative period, immediately after surgery, and postoperatively at 6 weeks. VAS is sufficiently reliable for being used to assess acute pain [3]. Any other significant complications in the intraoperative/ postoperative period were noted. We emphasized on the radiological outcome because we firmly believe that clinical improvement of radiculopathy depends on the decompression achieved and does not change much with the type of implant used to achieve fusion.

Radiological assessments were performed using preoperative cervical spine $\mathrm{X}$-rays and magnetic resonance images along with screening of the rest of the spine. Anteroposterior (AP) and lateral views with the neck in neutral position were included. All the X-rays were obtained in the standing position. X-rays immediately after surgery were obtained on the second day after removing the drain. This included an AP view and a lateral view in the neutral position. At 3 months, 6 months, 1 year, and 2 year follow-ups, AP and lateral views in flexion, extension, and neutral position were assessed. The radiological criteria included motion segment height, adjacent disc height (upper and lower), adjacent segment bone formation, segmental Cobb's angle, C2-7 Cobb's angle, implant subsidence, peri-implant lucencies, implant breakage, and pseudoarthrosis. Fusion was assessed by bridging bone formation and flexion-extension lateral views. No bridging bone or more than a $2^{\circ}$ change in angulation in flexion-extension views indicated pseudoarthrosis.

All patients were operated on by the senior author. The right-sided Smith-Robinson approach was used. After exposure level was confirmed with an image intensifier, discectomy was performed with the aid of a microscope. Posterior longitudinal ligament was cut in all cases to achieve complete decompression. Endplate was prepared, 
and an appropriate size trial implant was inserted and checked using an image intensifier. We used Zero-P (Synthes GmbH Switzerland, Oberdorf, Switzerland) in all the patients. Required adjustments were done, screw holes were made, and the original implant was inserted. We did not put the patients in skull traction at the time of surgery, but manual traction by an anesthetist was required in a few patients.

Statistical analysis was done using paired $t$ test. Followups were scheduled at 6 weeks, 3 months, 6 months, 1 year, and 2 years.

\section{Results}

Among the 37 patients recruited, 3 were lost for followup. Of the remaining patients, 34 were available for the 2 -years follow-up. There were 26 males and 8 females with an average age of 42 years (range, $26-58$ years). The most common level operated on was C5-6 in 16 patients, followed by C6-7 in 14 patients, C4-5 in 3 patients, and C3-4 in one patient.

Clinical improvement was excellent, with VAS scores improving from 7.4 to 0 for radiculopathy and 2.0 to 0.6 for neck pain. No patient had any symptoms of radiculopathy at the 2-year follow-up, but 4 patients complained of occasional neck pain. Because the neck pain was not significant enough for further investigation, they were not evaluated further. None of the patients had wound healing problems or change in voice. Mild dysphagia, which improved within 3 weeks, occurred in 8 patients.
Motion segment height improved by an average of $11.33 \%(p<0.001)$ based on the X-rays obtained immediately after surgery compared with that based on those obtained preoperatively. Maximum improvement noted was $19.75 \%$ in one case, and minimum improvement noted was $1 \%$. The median stood at $12.28 \%$. But this improvement in the motion segment height was not maintained in the follow-up. At the 2-year follow-up, there was an average loss of $7.03 \%(p<0.001)$ from the postoperative height. Maximum height noted was $19 \%$ and minimum noted was $3.39 \%$, with a median at $5.95 \%$. These results show that there some implant subsidence happens with time. The amount of loss of motion segment height indirectly corresponds to the amount of implant subsidence.

Adjacent segment disc space heights were compared preoperatively and at the 2-year follow-up. The average difference was $0.08 \%(p=0.8)$ in the upper disc space height, which was insignificant, and $0.16 \%(p<0.001)$ in the lower disc space height, which was significant. Not much information was available at 2 years, and we feel that further long-term studies are required to discover any adjacent segment changes.

Segmental and cervical Cobb's angulations were measured. Segmental data showed a preoperative average of $0.88^{\circ}$ of kyphosis and $5.59^{\circ}$ of lordosis as a postoperative average. This average improvement of $6.47^{\circ}$ was significant $(p<0.001)$. At the 2 -years follow-up, the improvement gained in the immediate postoperative period showed a significant loss (Fig. 1). The average 2-year segmental Cobb's was $1.38^{\circ}$ kyphosis, i.e., loss of $7.05^{\circ}(p<0.001)$

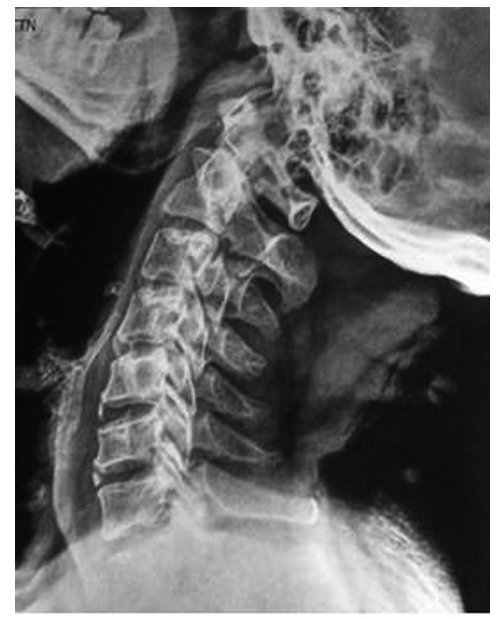

Preoperative

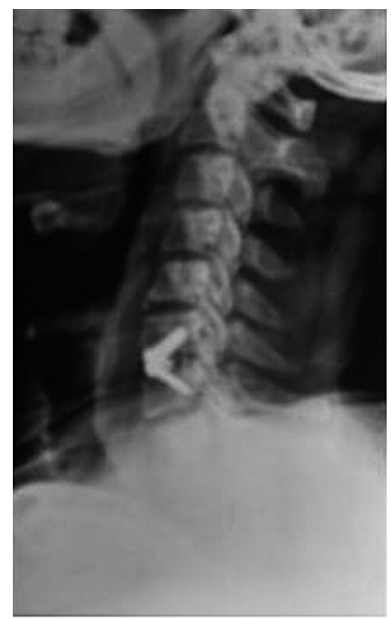

Immediate postoperative

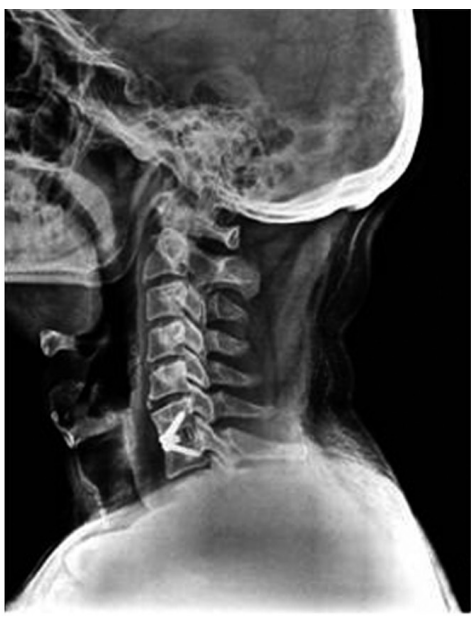

2 Year postoperative

Fig. 1. Anterior cervical discectomy and fusion at C6-7 using Zero-P cage screw construct. Note that at the 2-year follow-up, the improvement gained in the period immediately after surgery was significantly lost. 
from the postoperative status. Preoperatively, segmental kyphosis was present in 7 patients, and 3 patients had neutral alignment. Based on neutral alignment, we included patients with $-1^{\circ}$ and $+1^{\circ}$ Cobb's angles. However, postoperatively, a good correction was achieved in all these patients and none of them had localized kyphosis. Two patients had neutral alignment. This reversed at the last follow-up, with 8 patients developing localized kyphosis and 4 patients losing their lordosis to neutral alignment. Fig. 2 shows these details in a diagrammatic manner.

Cervical lordosis was measured from lower end plate of $\mathrm{C} 2$ to lower end plate of C7/C6 based on the X-rays. Preoperative average was $11.59^{\circ}$ of lordosis, which showed slight improvement to $14.88^{\circ}$ in the X-rays obtained immediately after surgery; the difference was insignificant $(p=0.164)$. Maximum improvement was observed at the 2 -year follow-up, with an average of $22.59^{\circ}$, implying a significant improvement of $11^{\circ}$ from the preoperative value $(p<0.001)$. Although 1 patient had kyphosis and 2 had neutral alignment in the preoperative period, they improved, and at the final follow-up, all patients were lordotic. Fig. 3 provides the details.

There was one patient who developed adjacent segment bone formation. There was a small osteophyte in the adjacent level in his preoperative X-ray itself, which increased in size at the 2-year follow-up. Hence, we believe that this cannot be attributed to the implant. Three patients showed bone formation anterior to the implant. This was seen in patients in whom the implant was placed well inside the disc space (Fig. 4). These patients also had more implant subsidence compared with others. In two patients, the implant was protruding by $2 \mathrm{~mm}$ due to a small AP diameter of vertebrae. This is a problem that can be avoided if the AP diameter of the vertebrae is preoperatively calculated. For the screws to have a good hold, accurate implant placement is important. Both these patients were female

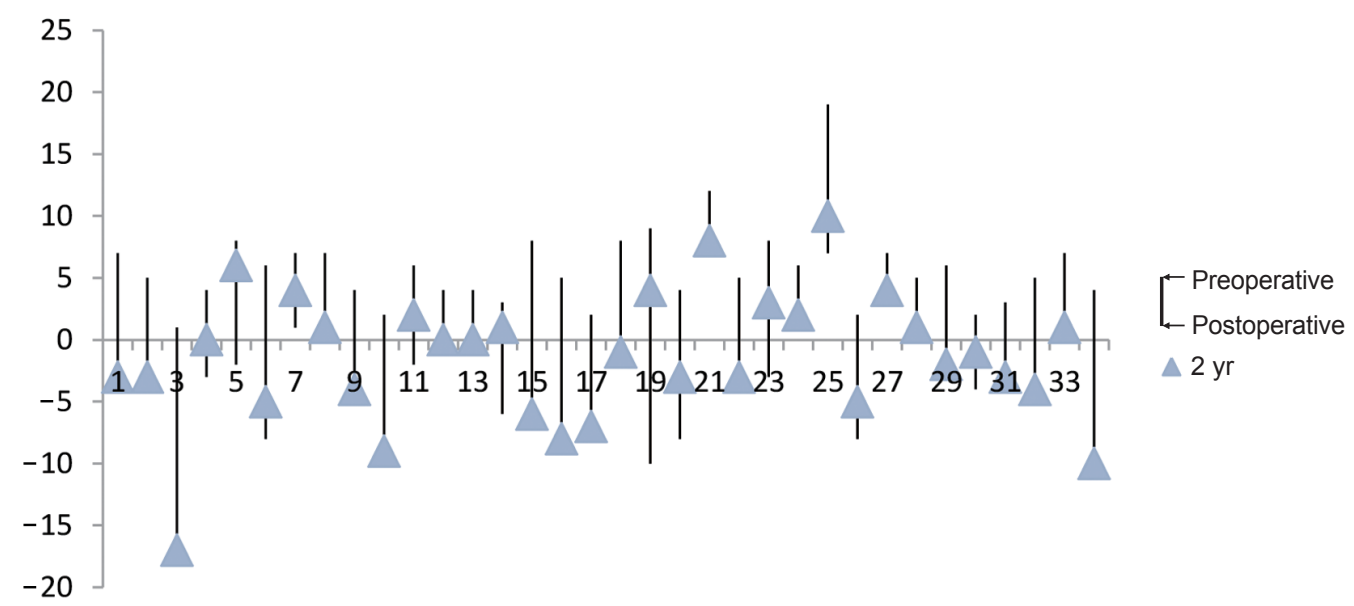

Fig. 2. The distribution of segmental angle change from the preoperative period through postoperative to the final follow-up. Y-axis represents the angle in degrees and $X$-axis shows the number of patients.

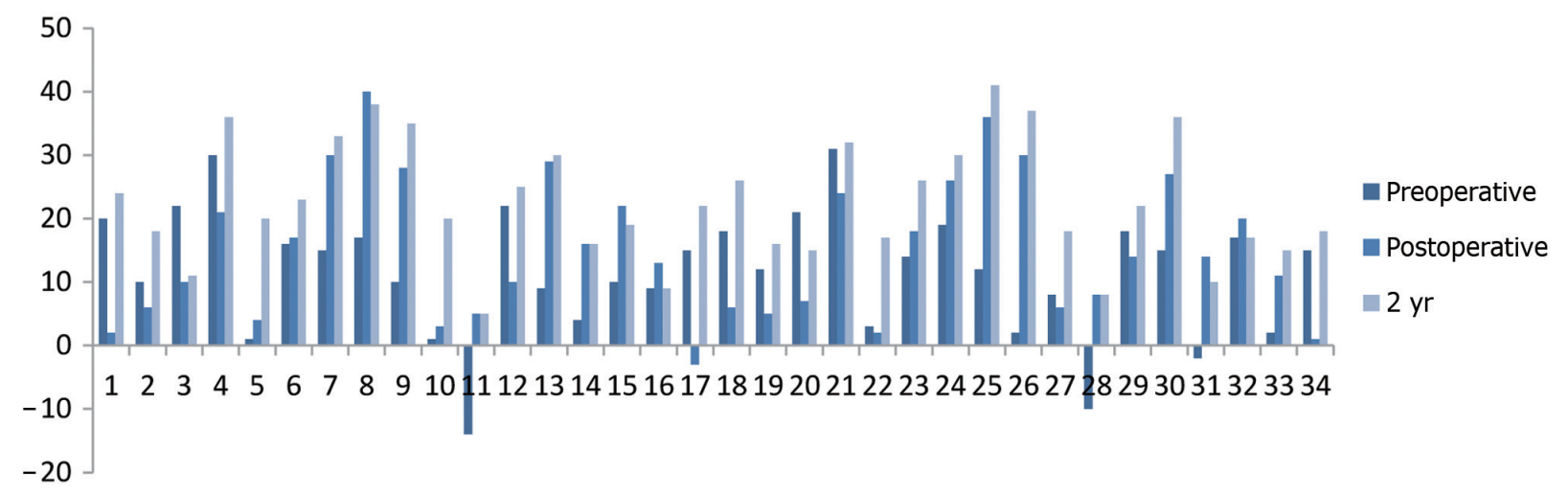

Fig. 3. The progressive improvement in cervical lordosis. $Y$-axis represents the angle and $X$-axis represents patients. 


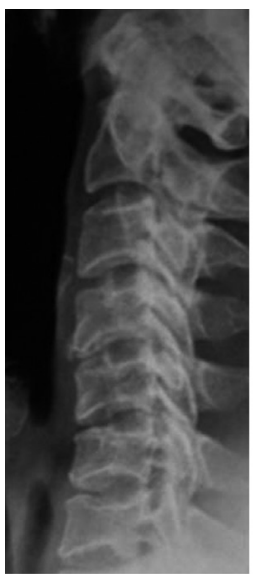

Preoperative

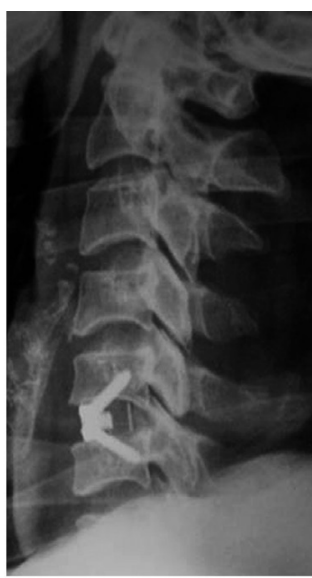

Immediate postoperative

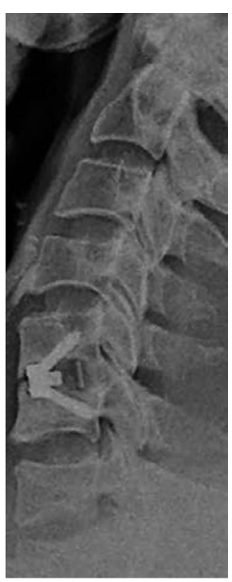

2 Year follow-up
Fig. 4. C5-6 anterior cervical discectomy and fusion with Zero-P cage screw construct. This patient showed bone formation anterior to the implant, which was placed well inside the disc space.

and thinly built. In another patient in whom implant was placed properly based on the X-ray obtained immediately after surgery, there was mild protrusion at the 2-year follow-up. The patient was completely asymptomatic. In the AP view, of the 34 implants, 21 showed some amount of angulation in the coronal plane, which persisted at the follow-up. No patients had implant loosening, pseudoarthrosis, or implant breakage. None of the patients had infection, implant migration, or a neurological deficit. Although these radiological problems persisted, no patient had any clinical symptoms. No resurgeries were performed.

\section{Discussion}

ACDF provides excellent results in cervical radiculopathy and myelopathy. These newer zero-profile implants seem to have the advantage of providing good fusion with fewer complications compared with anterior cervical plates. However, biomechanical stability is a problem with standalone cages. Stand-alone interbody cages in the cervical spine have shown inferior stability compared with plate fixation in flexion/extension and rotation. Lateral bending was the only movement when it was comparable with plate fixations. Moreover, the stand-alone cage showed more movement at the operated disc level compared with that in the normal spine [4]. Scholz et al. [5] did biomechanical testing on zero-profile implants with 24 cadavers. They showed that the anchored spacer provided a biomechanical stability similar to that provided by the estab- lished anterior fusion technique using an anterior plate plus cage. They supported progression to clinical trials using the cervical anchored spacer as a stand-alone implant [5]. Zero-P implants have a locking plate-screw interface, which holds the screw cage construct stable. The plate has an internal screw thread that engages with the outer screw thread located in the head of the screw. This makes the implant constrained and gives an angle-stable screw fixation [6].

When using a stand-alone cage system, subsidence of the implant is a problem that cannot be avoided. When stand-alone cages were compared with plate fixation, subsidence occurred in $32.3 \%$ of patients with cages and $9.7 \%$ of those with plate fixation, but the clinical outcomes were similar for both treatment groups [7]. An outcome analysis of Zero-P implants showed no implant subsidence and good fusion at the 3-month follow-up [8]. Compression to some extent at the operated site increases the chance of fusion and indirectly decreases the chance of implant failure. It has been reported that improvement in cervical lordosis is more important clinically in the long term than the impact of cage subsidence [9]. We found implant subsidence in all our patients. The average loss of motion segment height was $7.03 \%$. In few cases, this subsidence may leave the implant a little protruded. However, although as previously reported, this does not have an adverse clinical impact, a long-term follow-up is required before a final statement can be made.

Maintenance of cervical lordosis is more important for a good long-term clinical outcome. Rigid plate fixations when compared to dynamic plate fixations have shown better maintenance of lordosis achieved at the time of surgery. A comparative study on dynamic vs. rigid plate fixation has shown a significantly higher loss of segmental lordosis that did not influence the clinical outcome at the 2-year follow-up [10]. Whether the zero-profile implants behave like dynamic/rigid plates is controversial. Early follow-up outcomes of zero-profile implants showed that they could improve and maintain cervical lordosis and disk height [11]. It has been reported that Zero-P implants show advantages of both rigid and dynamic plate designs and avoid their implant-associated complications [8]. We differ slightly in this regard; we show that Zero-P implants behave more like a dynamic plate with loss of segmental lordosis, segmental height, and subsidence, thereby increasing the chances of fusion. Although there is loss of segmental lordosis, cervical lordosis is improved in most 
patients, which is much more important in the long term. This overall increase in cervical lordosis over few years is possibly due to the compensatory increase in range of motion at all the other levels for the loss at the segmental level [12].

Once a motion segment is fused, some changes in the adjacent segments are expected. Biomechanical testing using seven human cadavers showed the compensatory mechanisms by the spine to regain the motion lost in the fused segment, wherein maximum compensation occurred at the adjacent levels with significant increases at the level above for C3-4 and C4-5 fusions and below for C5-6 and C6-7 fusions during both flexion and extension [13]. In a short-term follow-up comparative study on ACDF vs. disc arthroplasty, 3.5 times more adjacent segment changes in fusion surgeries were observed; this study assessed range of motion, disc space angle, intervertebral height at the operated site, and adjacent levels [14]. We did not find any major changes in the adjacent segment disc height at the 2-year follow-up. Long-term studies are definitely warranted. However, in the long term, the natural degenerative process also plays a role in causing adjacent segment changes [15].

Adjacent level ossification is a known complication with anterior cervical plates. A review article reported a $41 \%-$ $64 \%$ risk of development of adjacent level ossification in ACDF, wherein the most important risk factor was the use of instrumentation and the plate-to-disc distance; furthermore, the surgical procedure type (corpectomy vs. discectomy and fusion) did not show statistical significance, and it was recommended that the surgeon should make every effort to keep the plate as far as possible from the adjacent disc [16]. Coated distance of $5 \mathrm{~mm}$ from the adjacent disc is reported to be safe for the upper end of the plate to rest on the bone [17]. This is one of the complications where zero-profile implants have a clear advantage over plate fixations. When using these implants there is decreased periosteal stripping and decreased soft tissue damage in the anterior cervical region. In our study, there also was one case of adjacent level ossification in the upper adjacent level, but it cannot be attributed to the implant as preoperative X-ray also showed ossification changes.

Dysphagia is one of the most discussed complications in anterior cervical spine surgery. Some of the theories on the etiology of postoperative dysphagia are postoperative soft-tissue edema, postoperative hematoma, and adhesion formations around the implants [18]. A correlation between the plate thickness and dysphagia rate, with decreased dysphagia with thinner plates has been observed [19]. With zero-profile implants, the incidence of postoperative dysphagia is shown to be infrequent. However, early postoperative dysphagia is not a complication related to anterior surgeries alone; it is also associated with posterior cervical surgeries. Dysphagia on a postoperative video fluoroscopic swallow evaluation was observed in $47 \%$ of anterior cervical surgeries and $21 \%$ cases of posterior cervical surgeries; this study reported an age of $>60$ years to be the only risk factor [20]. A cadaver study comparing retraction pressure during anterior cervical plate surgery and cervical disc replacement at the C5-6 level showed significantly greater pressures in the esophagus due to retraction with plate surgery regardless of whether it is a 3-level/single-level plate; cervical disc replacement seemed to require less retraction and thus reduced intraesophageal pressures when compared to plating [21]. This advantage with disc replacement can be extrapolated to zero-profile implants as the exposure required is the same for both. Here 8 patients developed dysphagia in the early postoperative period possibly due to postoperative soft tissue edema and the regional eating habits (dry chapattis and rotis), which cannot be attributed to the implant. This resolved by 3 weeks, and no long-term complications were observed.

Pseudoarthrosis is a common complication in uninstrumented fusions. In single-level discectomy without instrumentation, $11 \%$ pseudoarthrosis was observed [22]. In comparison to plate fixations, stand-alone cage implants had higher chances of pseudoarthrosis with requirement for revision surgery in $10.5 \%$ of stand-alone cages vs. none in plate fixation cases [8]. In a short-term follow-up with zero-profile implants, good fusion at the 3-month followup was observed [9]. A study reported good fusion in all patients at a minimum follow-up of 6 months [7]. We also found solid fusion in all our patients postoperatively at 6 months. We believe that implant subsidence seen in these patients also aids good bone formation.

Implant-related complications are quite frequently reported with plate fixations. A 5-11-year follow-up showed 9.9\% implant related complications but no requirement of revision surgery for implant failure [23]. In 2 cases of fracture of the implant, it was reported that improper contouring of the plate causing microstructural damage may have created a weak point and contributed to hardware failure [24]. Esophageal perforation by the screws of the 
anterior cervical plate is also a known complication. One case of asymptomatic esophageal perforation where a dislodged screw was found at 1 year has been reported [25]. Two technical problems with plates, positioning of the plate and the screw depth, have been previously reported; Although many improvements were reported, there is no foolproof technique for positioning the plate in the exact position [26]. Screw depth is also a persisting problem, although with locking plates available now, this is sorted to some extent. Positioning and screw depth are not major issues with zero-profile implants because we place them in the prepared disc space and because they comprise locking screws. However, proper coronal placement of these implants is challenging. Of our 34 patients, 21 had some angulation in the coronal plane despite taking adequate precautions for accurate placement.

\section{Conclusions}

Zero-profile cage screw devices provide good fusion and cervical lordosis with fewer complications. However, they are not capable of maintaining segmental lordosis and allow significant amount of motion segment collapse. We also recommend caution when using these in patients with short AP vertebral body lengths.

\section{Conflict of Interest}

No potential conflict of interest relevant to this article was reported.

\section{ORCID}

Saumyajit Basu: 0000-0001-7979-8745

\section{References}

1. Korinth MC. Treatment of cervical degenerative disc disease: current status and trends. Zentralbl Neurochir 2008;69:113-24.

2. Kuijper B, Tans JT, Beelen A, Nollet F, de Visser M. Cervical collar or physiotherapy versus wait and see policy for recent onset cervical radiculopathy: randomised trial. BMJ 2009;339:b3883.

3. Bijur PE, Silver W, Gallagher EJ. Reliability of the visual analog scale for measurement of acute pain. Acad Emerg Med 2001;8:1153-7.
4. Shimamoto N, Cunningham BW, Dmitriev AE, Minami A, McAfee PC. Biomechanical evaluation of stand-alone interbody fusion cages in the cervical spine. Spine (Phila Pa 1976) 2001;26:E432-6.

5. Scholz M, Reyes PM, Schleicher P, et al. A new standalone cervical anterior interbody fusion device: biomechanical comparison with established anterior cervical fixation devices. Spine (Phila Pa 1976) 2009; 34:156-60.

6. Scholz M, Schnake KJ, Pingel A, Hoffmann R, Kandziora F. A new zero-profile implant for stand-alone anterior cervical interbody fusion. Clin Orthop Relat Res 2011;469:666-73.

7. Song KJ, Taghavi CE, Lee KB, Song JH, Eun JP. The efficacy of plate construct augmentation versus cage alone in anterior cervical fusion. Spine (Phila Pa 1976) 2009;34:2886-92.

8. Azab W, Abdel-Razek M, Ali A, et al. Outcome evaluation of a zero-profile implant for anterior cervical diskectomy with fusion. Turk Neurosurg 2012;22: 611-7.

9. Wu WJ, Jiang LS, Liang Y, Dai LY. Cage subsidence does not, but cervical lordosis improvement does affect the long-term results of anterior cervical fusion with stand-alone cage for degenerative cervical disc disease: a retrospective study. Eur Spine J 2012;21: 1374-82.

10. Pitzen TR, Chrobok J, Stulik J, et al. Implant complications, fusion, loss of lordosis, and outcome after anterior cervical plating with dynamic or rigid plates: two-year results of a multi-centric, randomized, controlled study. Spine (Phila Pa 1976) 2009;34:641-6.

11. Miao J, Shen Y, Kuang Y, et al. Early follow-up outcomes of a new zero-profile implant used in anterior cervical discectomy and fusion. J Spinal Disord Tech 2013;26:E193-7.

12. Auerbach JD, Anakwenze OA, Milby AH, Lonner BS, Balderston RA. Segmental contribution toward total cervical range of motion: a comparison of cervical disc arthroplasty and fusion. Spine (Phila Pa 1976) 2011;36:E1593-9.

13. Schwab JS, Diangelo DJ, Foley KT. Motion compensation associated with single-level cervical fusion: where does the lost motion go? Spine (Phila Pa 1976) 2006;31:2439-48.

14. Kim SW, Limson MA, Kim SB, et al. Comparison of radiographic changes after $\mathrm{ACDF}$ versus Bryan disc 
arthroplasty in single and bi-level cases. Eur Spine J 2009;18:218-31.

15. Goffin J, Geusens E, Vantomme N, et al. Long-term follow-up after interbody fusion of the cervical spine. J Spinal Disord Tech 2004;17:79-85.

16. Kim HJ, Kelly MP, Ely CG, Dettori JR, Riew KD. The risk of adjacent-level ossification development after surgery in the cervical spine: are there factors that affect the risk? A systematic review. Spine (Phila Pa 1976) 2012;37(22 Suppl):S65-74.

17. Park JB, Cho YS, Riew KD. Development of adjacentlevel ossification in patients with an anterior cervical plate. J Bone Joint Surg Am 2005;87:558-63.

18. Fountas KN, Kapsalaki EZ, Nikolakakos LG, et al. Anterior cervical discectomy and fusion associated complications. Spine (Phila Pa 1976) 2007;32:2310-7.

19. Lee MJ, Bazaz R, Furey CG, Yoo J. Influence of anterior cervical plate design on dysphagia: a 2-year prospective longitudinal follow-up study. J Spinal Disord Tech 2005;18:406-9.

20. Smith-Hammond CA, New KC, Pietrobon R, Curtis DJ, Scharver $\mathrm{CH}$, Turner DA. Prospective analysis of incidence and risk factors of dysphagia in spine surgery patients: comparison of anterior cervical, posterior cervical, and lumbar procedures. Spine (Phila $\mathrm{Pa}$
1976) 2004;29:1441-6.

21. Tortolani PJ, Cunningham BW, Vigna F, Hu N, Zorn $\mathrm{CM}$, McAfee PC. A comparison of retraction pressure during anterior cervical plate surgery and cervical disc replacement: a cadaveric study. J Spinal Disord Tech 2006;19:312-7.

22. Wright IP, Eisenstein SM. Anterior cervical discectomy and fusion without instrumentation. Spine (Phila Pa 1976) 2007;32:772-4.

23. Yue WM, Brodner W, Highland TR. Long-term results after anterior cervical discectomy and fusion with allograft and plating: a 5- to 11-year radiologic and clinical follow-up study. Spine (Phila Pa 1976) 2005;30:2138-44.

24. Yen CP, Hwang TY, Wang CJ, Howng SL. Fracture of anterior cervical plate implant: report of two cases. Acta Neurochir (Wien) 2005;147:665-7.

25. Pompili A, Canitano S, Caroli F, et al. Asymptomatic esophageal perforation caused by late screw migration after anterior cervical plating: report of a case and review of relevant literature. Spine (Phila $\mathrm{Pa}$ 1976) 2002;27:E499-502.

26. Gassman J, Seligson D. The anterior cervical plate. Spine (Phila Pa 1976) 1983;8:700-7. 